\title{
Synthesis of Polycrystalline Ruddlesden-Popper Organic Lead Halides and Their Growth Dynamics
}

\author{
${ }^{1}$ Raphael Fernando Moral, ${ }^{1}$ Luiz Gustavo Bonato, ${ }^{1} J o s e ́$ Carlos Germino, ${ }^{1,2}$ Willian Xerxes Coelho Oliveira, ${ }^{3,4}$ Rupini \\ Kamat, ${ }^{3}$ Junwei Xu, ${ }^{3}$ Christopher Tassone, ${ }^{5}$ Samuel D. Stranks, ${ }^{3}$ Michael Toney and ${ }^{1}$ Ana Flávia Nogueira. \\ ${ }^{1}$ Laboratório de Nanotecnologia e Energia Solar, Chemistry Institute, University of Campinas, Campinas, Sao Paulo, \\ Brazil. \\ ${ }^{2}$ Departamento de Química, Institudo de Ciências Exatas, Universidade Federal de Minas Gerais, Belo Horizonte, \\ Minas Gerais, Brazil. \\ ${ }^{3}$ Stanford Synchrotron Radiation Lightsource, SLAC National Accelerator Laboratory, Menlo Park, CA 94025, Unit- \\ ed States of America. \\ ${ }^{4}$ Department of Applied Physics, Stanford University, Stanford, CA 94305, United States of America. \\ ${ }^{5}$ Cavendish Laboratory, University of Cambridge, Cambridge, $\mathrm{CB}_{3}$ oHE, United Kingdom.
}

Supporting Information Placeholder

\begin{abstract}
Ruddlesden-Popper organic lead halides (RPOLHs) are in the material science spotlight due to their remarkable optoelectronic properties, tailorable compositions and improved ambient stability over their threedimensional perovskite analogues. In this report, we introduce a new synthesis method for RPOLHs with general composition $\mathrm{L}_{2}\left[\mathrm{FAPbI}_{3}\right]_{\mathrm{n}-1} \mathrm{PbI}_{4}(\mathrm{~L}=$ butylammonium $(\mathrm{BA})$ or benzylammonium (BZA), $\mathrm{FA}=$ formamidinium, and $n=1$ and 2 ). Our method yields polycrystalline materials useful for thinfilm fabrication for optoelectronics. Furthermore, we report the growth dynamics of $\mathrm{BA}_{2}\left[\mathrm{FAPbI}_{3}\right] \mathrm{PbI}_{4}$ using in situ small angle $\mathrm{X}$-ray scattering, revealing that the slabs grow within the first seconds of the reaction, and then self-assemble into bulk crystallites.
\end{abstract}

\section{INTRODUCTION}

With the recent rise in perovskite-based optoelectronic devices, many scientific achievements have been reported, comprising the many facets of these materials and the challenges to their application. ${ }^{1-5}$ Among the lead halide perovskites (LHPs), Ruddlesden-Popper organic lead halides (RPOLHs), an analogue class of materials (commonly referred to as $2 \mathrm{D}$-perovskites), are gaining increasing interest. In comparison with their $3 \mathrm{D}$ LHP analogues, RPOLHs present higher ambient stability and wider compositional variability. Recently, RPOLHs have been applied to passivate perovskite solar cells, ${ }^{6-8}$ and the pure RPOLH-based solar cells have achieved up to $16.5 \%$ efficiency ${ }^{9}$ and excellent ambient stability. ${ }^{10-12}$ Additionally, these materials have been applied in efficient $\mathrm{LEDs}^{13,14}$ and photodetector. ${ }^{15}$
A RPOLH crystalline phase can be visualized as a sliced $3 \mathrm{D}$ LHP along the crystallographic direction [ool] (or orthogonal coordinates) ${ }^{16,17}$ If the A-site cation is too large to fit in the cuboctahedral site of a LHP, it sits as a bridge, separating the planes perpendicular to the [ool] direction (Figure 1). The final structure is an assembly of $\left[\mathrm{PbX}_{6}\right]^{4-}$ octahedron slabs sandwiched between a bilayer of organic cationic species. This organic bilayer imparts enhanced stability of the optoelectronic devices, since it hinders the diffusion of water into the crystal lattice. ${ }^{6,18,19}$

The chemical formula of a RPOLH is written as $\mathrm{L}_{2}\left[\mathrm{APbX}_{3}\right]_{\mathrm{n}-1} \mathrm{PbI}_{4}$; where, $n$ is the number of octahedron layers in a single slab; $\mathrm{L}$ is a cationic species, usually an alkylammonium; $\mathrm{A}$ is the cation of the corresponding $\mathrm{APbX}_{3} \mathrm{LHP}$; and $\mathrm{X}$ is a halide. Therefore, depending on the value of $n$, these materials may present anisotropic quantum-confinement of the optical excitations. ${ }^{20-22}$ Moreover, RPOLHs present compositional dependence of the bandgap energy, which increases as the composition varies from iodide to chloride. Because of these properties and the tailorable composition, RPOLHs are promising materials for next generation optoelectronics. ${ }^{23,24}$

The preparation of RPOLHs is largely restricted to three classic approaches: spin-coating from precursor solutions directly onto a substrate; $;^{9,13,25-28}$ single crystal growth in highly acidic solutions (concentrated HX); ${ }^{1629-33}$ or colloidal nanocrystals (NCs) in the presence of longchain capping ligands. ${ }^{34-38}$ Although these three methods are highly important in different circumstances and uses, they present some drawbacks and limitations. For instance, thin films fabricated directly from precursor solutions limits control over the thickness of the phases (i.e., 
the values of $n) .^{9,13}$ By contrast, single crystal growth allows a finer control over the thickness of the slabs; however, these synthetic methods are rather expensive and aggressive. To date, NCs of these materials exhibit poor
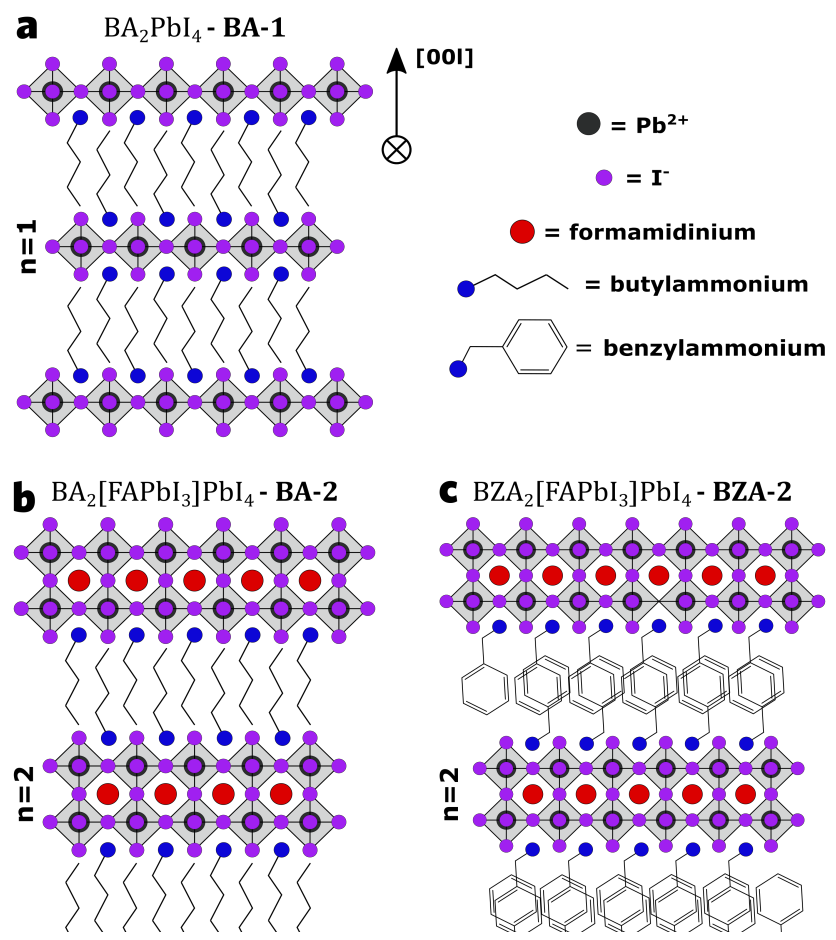

Figure 1. Illustration of the three different RPOLHs in this

work a) $\mathrm{BA}_{2} \mathrm{PbI}_{4}$, b) $\mathrm{BA}_{2}\left[\mathrm{FAPbI}_{3}\right] \mathrm{PbI}_{4}$, and c) $\mathrm{BZA}_{2}\left[\mathrm{FAPbI}_{3}\right] \mathrm{PbI}_{4}$.
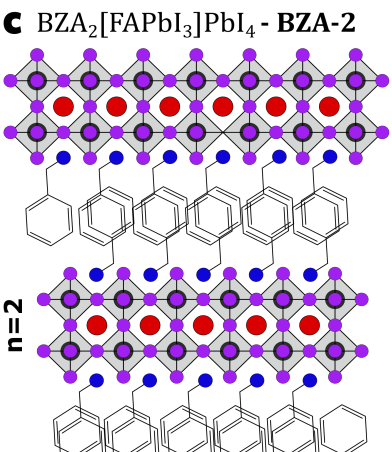

stability and difficult processability; during the process of purification, they easily degrade due to ligand removal. ${ }^{39,40}$ Therefore, other preparation methods, allowing easy processability and thin-film fabrication, are desirable.

In this work, we present a new strategy for lowtemperature, solution-process synthesis of polycrystalline $\mathrm{L}_{2}\left[\mathrm{FAPbI}_{3}\right]_{\mathrm{n}-1} \mathrm{PbI}_{4}$ materials; where, $\mathrm{FA}=\left[\mathrm{CH}\left(\mathrm{NH}_{2}\right)_{2}\right]^{+}$ (formamidinium), $\mathrm{L}=$ n-butylammonium $\left(\mathrm{BA}=\mathrm{C}_{4} \mathrm{H}_{9}-\right.$ $\mathrm{NH}_{3}^{+}$) or benzylammonium (BZA $=\mathrm{C}_{6} \mathrm{H}_{5}-\mathrm{CH}_{2}-\mathrm{NH}_{3}{ }^{+}$), and $n$ is 1 and 2 . These materials can be easily processed for thin-film fabrication, making them suitable for optoelectronic applications. Some reports describe the preparation of thin-films by dissolving single crystals and spincasting their solutions; two of which apply them to efficient optoelectronic devices. ${ }^{11,14,31}$ Our synthesis allows the same fabrication approach, without the need of the demanding single-crystal synthesis.

Additionally, we present important aspects of the growth dynamics of the $\mathrm{BA}_{2}\left[\mathrm{FAPbI}_{3}\right] \mathrm{PbI}_{4}$ material using in situ small angle $\mathrm{X}$-ray scattering. Our findings shed light on the growth mechanism of this type of layered lead halide materials in solution and may be extended to a more general comprehension of the growth dynamics of analogue materials.

\section{SYNTHESIS}

Briefly, the synthesis of these materials consists of a rapid mixture of two solutions: a toluene solution of $\mathrm{PbI}_{2}$ with butyric acid and the corresponding amine, and a toluene solution of FA-acetate (for $n=2$ materials) or butylammonium iodide, both with butyric acid. Upon the mixture of these solutions, the solid materials are readily formed, except for the butylamine-based one that requires heat $\left(70{ }^{\circ} \mathrm{C}\right)$ - see Supplementary Material for details. For simplicity, the solid with $n=1$ will be denoted as BA-1 (Figure 1a), and the other two materials with $n=2$ will be denoted as BA-2 and BZA-2 (Figures $1 \mathrm{~b}$ and $1 \mathrm{c}$, respectively).

In general, this synthetic approach of RPOLHs relies on the high excess of ammonium cations relative to lead iodide and/or formamidinium (25-fold amine excess). To assure the formation of ammonium species, we use a large ratio acid:amine (4:1 v/v, respectively). The high concentration of ammonium species grants the conditions for an anisotropic growth of the RPOLHs, as observed in previous reports. ${ }^{41,42}$ The mechanism for this anisotropic growth is not fully understood; we herein use in situ small angle X-ray scattering (SAXS) measurements to elucidate aspects of these growth dynamics.

Briefly, we probed the reaction as it took place in beamline 1-5 at SSRL; we collected data before and after the mixture of the two precursor solutions of BA-2. Figures $2 \mathrm{a}, \mathbf{2 b}$, and $2 \mathrm{c}$ show the real-time SAXS data collected during this reaction. The injection of FA-solution lasted less than 1 second, and it took place between 3 - 5 seconds of data collection (gray and purple curves in Figure 2a). Hence, in the first 5 seconds of collection, all the precursors were already present in the reactor. In the following 10 seconds, the scattering intensity continues to increase until it reaches its maximum (quadrant 1 in Figure 2a); concomitantly, the scattering intensity coming from higher $Q$ values decreases at approximately the same rate (quadrant 2 in Figure 2a). We attribute these changes to the growth of $\mathrm{BA}_{2}\left[\mathrm{FAPbI}_{3}\right] \mathrm{PbI}_{4}$ slabs and consumption of the reaction precursors, respectively. Interestingly, between 10 - 15 seconds, the slabs start to stack, and a discrete diffraction peak emerges with the maximum at 0.29 $\AA^{-1}$ (Figure $2 \mathrm{a}$ - cyan curve). In the next 40 minutes of reaction, this diffraction peak sharpens, shifting the maximum to $0.32 \AA^{-1}$, and its intensity rises (Figures $2 \mathrm{~b}$ and 2c).

From these SAXS data, we observe that the BA-2 slabs form within the first 10 seconds and, in the sequence, the slabs self-assemble into the bulk material. The excess of ammonium cations allows a fast, anisotropic growth of the layers. The diffraction peak in the position $0.32 \AA^{-1}$ comes from the planes $\{020\}$ of the bulk BA-2 material $(\mathrm{d}$ = $19.6 \AA$ ), confirmed by powder X-ray diffraction (PXRD) - in Figure $3 b$. In a simplistic view, the peak width is inversely proportional to the crystallite size, ${ }^{43}$ and so we estimate the rate of the self-assembly of the platelets from analyzing the full-width-at-half-maximum (FWHM) sharpening as a function of time. The variation of the FWHM with time follows the equation: 
conditions: under white light, UV-light $(365 \mathrm{~nm})$, and
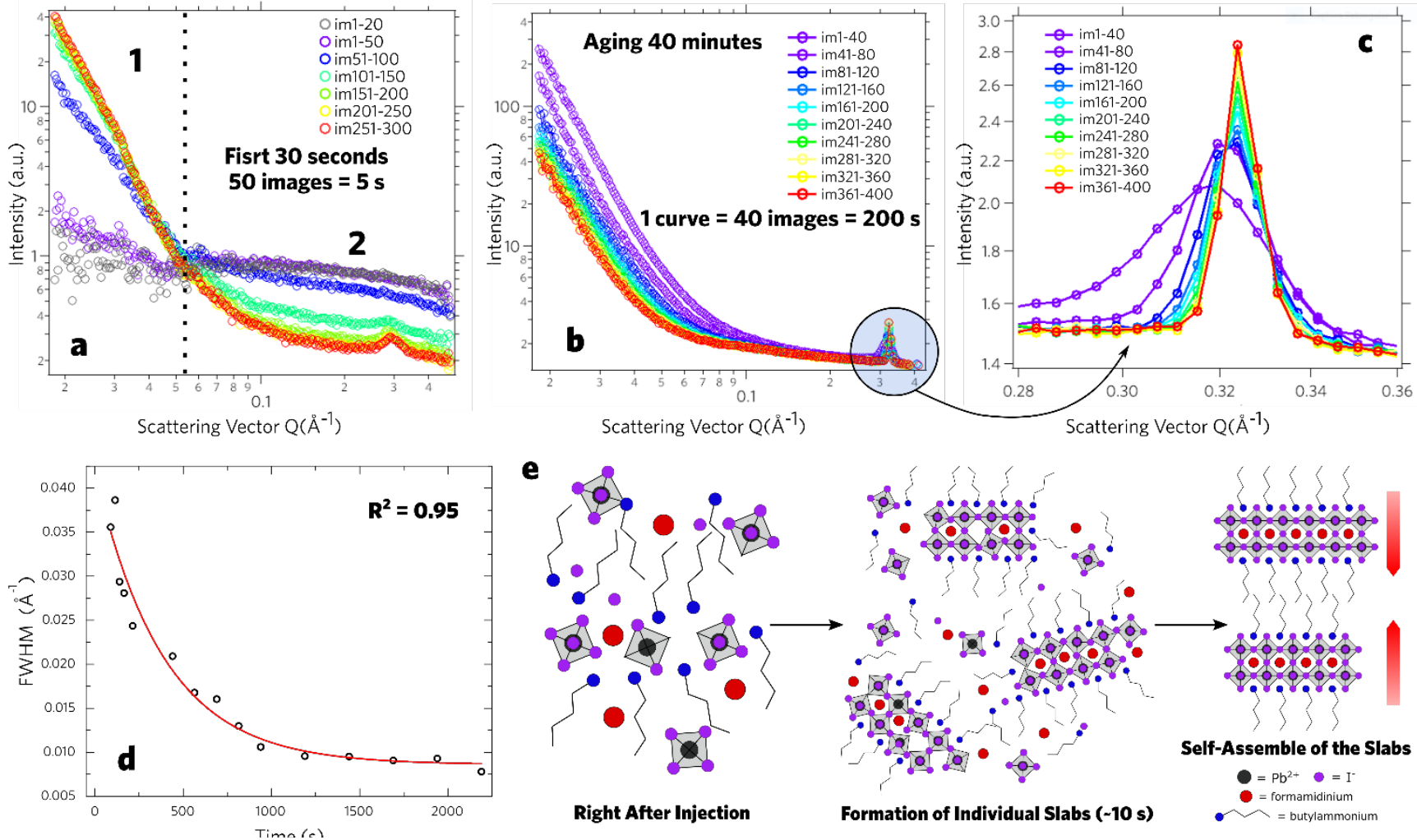

Figure 2. Real-time SAXS data from the BA-2 reaction a) SAXS curves of the first 30 seconds of the reaction; b) SAXS curves of the following 40 minutes of the reaction; c) the diffraction peak from b emphasized; d) the FWHM as a function of time; and e) general scheme of the formation mechanism of BA-2.

$$
-\frac{d(F W H M)}{d t}=k \cdot(F W H M)
$$

whose solution is a monoexponential decay with a rate $k$. This relation is depicted in Figure 2d, and the rate calculated from the fitting was $2.6 \times 10^{-3} \pm 0.5 \times 10^{-3} \mathrm{~s}^{-1}$.

With this rate we were able to demonstrate that it takes the stacking of two slabs ca. 6 s (see Supporting Material for details). Figure ze summarizes the growth mechanism of BA-2. Although the formation of the slabs is fast, their self-assembly is slow and extends through the next 40 minutes of reaction. Finally, the shift in the maximum of the diffraction indicates that, in the beginning, some solvent molecules might be intercalated in between slabs; as the crystal grows, it excludes these molecules from the structure, shifting the diffraction to higher $Q$ value (smaller d-spacing).

An important aspect to consider regarding this synthesis is the possibility of formation of thicker QW-phases in the $n=2$ materials (i.e., $n>2$ ), since they form in solution with no stoichiometric amounts of their components. However, we only observe these higher $n$-phases in the PXRD pattern of BZA-2 (Figure 3c). For BA-2, SAXS and PXRD data show only diffractions from the $n=2$ phase. Nonetheless, higher $n$-phases in small domains, undetectable from PXRD, cannot be ruled out.

To verify post-synthetic formation of higher $n$-phases while mimicking ambient effects, we collected several diffraction patterns of the powder BA-2 in three different temperature (see Supporting Figure 1). Under illumination conditions, we observed no changes in the XRD patterns, nor the surgency of any other $n$-phase (Supporting Figures $1 \mathrm{~b}$ and $1 \mathrm{c}$ ). More surprising, we could not observe changes in the sample under heat until $120{ }^{\circ} \mathrm{C}$, revealing a surprising thermal stability. The only difference observed was a shift of the diffractions due to thermal dilatation (Supporting Figure id).

To further assess the thermal stability of these materials, we carried out thermogravimetric analysis (TGA), depicted in Supporting Figure 2. In short, the organic moieties are not easily lost in these materials (not before $\sim 200{ }^{\circ} \mathrm{C}$ ). Therefore, we expect that higher $n$-phases, both in BA-2 and BZA-2, come mostly from the synthesis, rather than from amine or iodine loss in ambient conditions.

\section{STRUCTURAL AND OPTICAL CHARACTERIZATION}

Figure 3 shows the synchrotron based PXRD of the three materials along with the results from the Rietveld refinement. The PXRD data of BA-1 and BA-2 (Figures za and $3 \mathrm{~b}$, respectively) present strong correlation with their Rietveld refinements without adding any other potential contaminant phases (e.g., $\mathrm{PbI}_{2}$ and other higher $n$ phases). Even when accounting these contaminants, for both materials, the refinements yielded only trace amounts of $\mathrm{PbI}_{2}$ in BA-1 $(<0.01 \%$ in mol). For BZA-2, 
the correlation between experimental PXRD and the
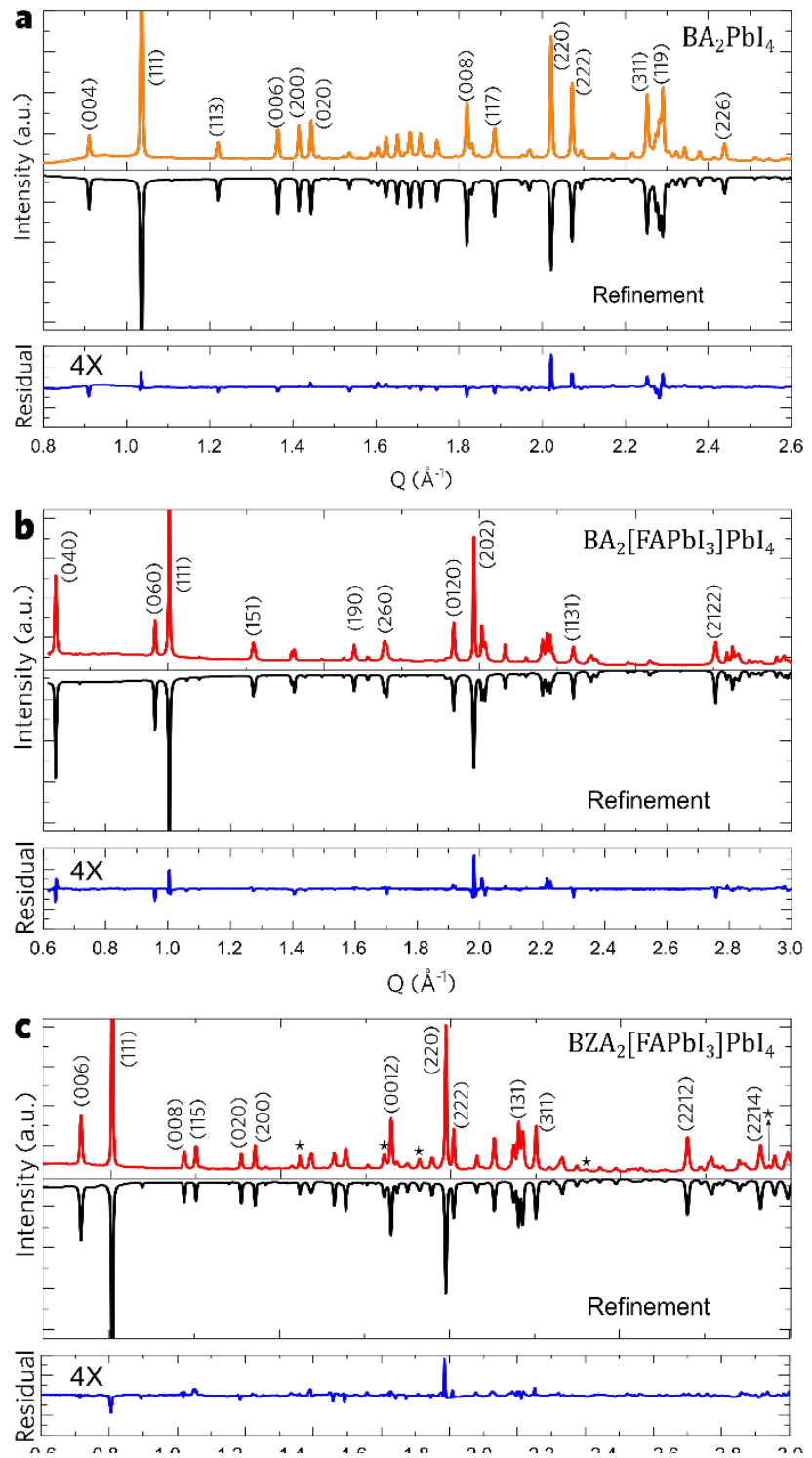

Figure 3. Synchrotron-based PXRD along with their respective Rietveld refinement curves (black) and residual plot from the refinement (blue) augmented four times of a) BA-1, b) BA-2, and c) BZA-2.

refinement using pure phase BZA-2 was not satisfactory (the asterisks in Figure $3 \mathrm{c}$ show peaks from other phases). For a stronger correlation, we needed to consider other $n$-phases ( $n$ from $1-5$ ), $\mathrm{PbI}_{2}$, and $\mathrm{FAPbI}_{3}$. The resulting composition obtained was as follows: BZA-2 = $0.66 ; \mathrm{PbI}_{2}=0.15 ; \mathrm{BZA}-1(n=1)=0.10$; and BZA-4 $(n=4)=$ o.o84 (molar fraction). These other materials found in BZA-2 powder form most likely due to its rougher formation pathway. During the synthesis, upon the mixture of the two precursor solutions, a dark brown solid is formed; as this suspension is heated at $70{ }^{\circ} \mathrm{C}$, it slowly turns into the red BZA-2 (see Supporting Figure 3).

For benchtop-PXRD measurements, these materials orient as one presses them on the sample holder. Hence, the generated diffraction patterns present highly intense Bragg reflections of the parallel planes along the [ool] direction ([oko] direction for BA-2) - see Supporting Figure 4. This orientation occurs due to the plaque-like morphology of the RPOLHs (Supporting Figure 5).

The absorption, measured by reflectance, and photoluminescence (PL) spectra, as well as the corresponding Tauc plots, of the three materials are depicted in Supporting Figure 6. The optical bandgap determined from the Tauc plots were as follows: $\mathrm{BA}-\mathbf{1}=\mathbf{2} .30 \mathrm{eV}, \mathrm{BA}-\mathbf{2}=\mathbf{2} .08 \mathrm{eV}$, and BZA-2 $=2.06 \mathrm{eV}$ (Supporting Figures 3b, 3d, and $3 \mathrm{f}$, respectively). BA-1 presents a sharp emission band at 520 $\mathrm{nm}$ that is characteristic of a free-exciton emission, presenting nearly no Stokes-shift from the absorption edge (Supporting Figure 3a). ${ }^{17,44}$ The other wider band, with maximum at $545 \mathrm{~nm}$, is quite unusual, and is similar to the single crystal emission reported by Mitzi. $^{29}$ BA-2 also presents two bands: the freeexcitonic emission $(579 \mathrm{~nm})$ and a lower energy one (Supporting Figure 3c). This lower energy band at 609 $\mathrm{nm}$ is consistent with a QW with $\mathrm{n}=3 \cdot{ }^{16,42}$ It is important to highlight the fact that thicker QWs, even in small concentrations, can effectively appear in the PL due to energy transfer from the thinner ones. In BA-2, it seems to be the case, although we do not observe any evidence of $n=3$ phases in its PXRD. BZA-2 presents an absorption spectrum very similar to BA-2, but its PL is highly affected by its impurities. The PL of BZA-2 clearly shows emissions from higher n-phases; in addition, this material also presents an emission band that is likely to be from selftrapped excitons (76o $\mathrm{nm}$ broad band), as previously observed for similar materials. ${ }^{15,45,46}$

Lastly, the thin-film properties of the three materials are quite similar to their powders (see Supporting Information for thin-film fabrication). BA-1 thin-film is highly emissive and shows the same optical features of the powder, except for the lower energy band in $545 \mathrm{~nm}$ (Supporting Figure $7 a$ ). BA-2 thin film (Supporting Figure $7 \mathrm{~b}$ ) is also emissive and shows similarities to the powder, with the second emission band shifted to lower energies (from 609 to $628 \mathrm{~nm}$ ). The BZA-2 thin film (Supporting Figure 7c) presents the same features of the powder.

\section{CONCLUSIONS}

In this work, we present an alternative synthesis to prepare polycrystalline RPOLHs. The simplicity of the synthesis and the absence of high boiling point solvents grant the easy processability of the products, making them cheap and appealing materials for optoelectronic applications. Furthermore, this method allows the preparation of other similar materials with a plethora of other compositions for future works (e.g., other organic acids, different organic spacers (amines), different A- and B-site cations, and other halogens). This synthetic approach can be used for thin-films fabrication for optoelectronic applications.

Our results with SAXS measurements reveal that, for BA-2, the $\left[\mathrm{PbX}_{6}\right]^{4-}$ octahedron slabs forms in the first seconds of the reaction, and then they self-assemble into bulk RPOLH crystallites. For the benzylamine-based ma- 
terial, the growth dynamics is different, and it requires heat $\left(70{ }^{\circ} \mathrm{C}\right)$. This difference is probably due to the steric hinderance caused by the aromatic backbone of benzylammonium cations; this observation might apply to other aromatic amines such as phenylethylamine (PEA) in a similar solution process synthesis.

\section{ASSOCIATED CONTENT}

\section{Supporting Information}

(Word Style "Section_Content"). A listing of the contents of each file supplied as Supporting Information should be included. For instructions on what should be included in the Supporting Information as well as how to prepare this material for publication, refer to the journal's Instructions for Authors.

The Supporting Information is available free of charge on the ACS Publications website.

brief description (file type, i.e., PDF)

brief description (file type, i.e., PDF)

\section{AUTHOR INFORMATION}

\section{Corresponding Author}

(Word Style "Section_Content"). Give contact information for the author(s) to whom correspondence should be addressed.

\section{ORCID}

Raphael F. Moral - oooo-0oo2-1844-4035.

\section{Present Addresses}

(Word Style "Section_Content"). +If an author's address is different than the one given in the affiliation line, this information may be included here.

\section{Author Contributions}

(Word Style "Section_Content"). ‡These authors contributed equally. (match statement to author names with a symbol, if applicable)

\section{Notes}

(Word Style "Section_Content"). The authors declare no competing financial interests.

Any additional relevant notes should be placed here.

\section{ACKNOWLEDGMENT}

Use of the Stanford Synchrotron Radiation Lightsource, SLAC National Accelerator Laboratory, is supported by the U.S. Department of Energy, Office of Science, Office of Basic Energy Sciences under Contract No. DE-ACo2-76SFoo515. The authors thank Laboratorio Nacional de Luz Sincrotron (LNLS) for providing beamtime at XRD2. RFM thanks the support from FAPESP - process numbers: 2016/02268-9 and 2017/11019-5. AFN and RFM gratefully acknowledge support from FAPESP (the Sao Paulo Research Foundation, Process 2017/11986-5) and Shell and the strategic importance of the support given by ANP (Brazil's National Oil, Natural Gas and Biofuels Agency) through the R\&D levy regulation. The powder X-ray diffraction, structure refinement, and SAXS inter- pretation were supported as part of the Center for Hybrid Organic Inorganic Semiconductors for Energy (CHOISE), an Energy Frontier Research Center Funded by the US Department of Energy, Office of Science, Basic Energy Sciences, under Award Number DE-AC36-o8Go28308. RVK acknowledges NSF fellowship.

\section{REFERENCES}

(1) Dunlap-Shohl, W. A.; Zhou, Y.; Padture, N. P.; Mitzi, D. B. Synthetic Approaches for Halide Perovskite Thin Films. Chem. Rev. 2019, 119 (5), 3193-3295.

(2) Xiao, M.; Huang, F.; Huang, W.; Dkhissi, Y.; Zhu, Y.; Etheridge, J.; Gray-Weale, A.; Bach, U.; Cheng, Y.-B.; Spiccia, L. A Fast Deposition-Crystallization Procedure for Highly Efficient Lead Iodide Perovskite Thin-Film Solar Cells. Angew. Chemie Int. Ed. 2014, 53 (37), 9898-9903.

Pellet, N.; Gao, P.; Gregori, G.; Yang, T.-Y.; Nazeeruddin, M. K.; Maier, J.; Grätzel, M. Mixed-Organic-Cation Perovskite Photovoltaics for Enhanced Solar-Light Harvesting. Angew. Chemie Int. Ed. 2014, 53 (12), 3151-3157.

(4) Saliba, M.; Matsui, T.; Domanski, K.; Seo, J.-Y.; Ummadisingu, A.; Zakeeruddin, S. M.; Correa-Baena, J.-P.; Tress, W. R.; Abate, A.; Hagfeldt, A.; et al. Incorporation of Rubidium Cations into Perovskite Solar Cells Improves Photovoltaic Performance. Science 2016, 354 (6309), 206209.

Szostak, R.; Marchezi, P. E.; Marques, A. dos S.; da Silva, J. C.; de Holanda, M. S.; Soares, M. M.; Tolentino, H. C. N.; Nogueira, A. F. Exploring the Formation of Formamidinium-Based Hybrid Perovskites by Antisolvent Methods: In Situ GIWAXS Measurements during Spin Coating. Sustain. Energy Fuels 2019.

(6) Lee, J.-W.; Dai, Z.; Han, T.-H.; Choi, C.; Chang, S.-Y.; Lee, S.-J.; De Marco, N.; Zhao, H.; Sun, P.; Huang, Y.; et al. 2D Perovskite Stabilized Phase-Pure Formamidinium Perovskite Solar Cells. Nat. Commun. 2018, 9 (1), 3021. de Holanda, M. S.; Szostak, R.; Marchezi, P. E.; Duarte, L. G. T. A.; Germino, J. C.; Atvars, T. D. Z.; Nogueira, A. F. In Situ 2D Perovskite Formation and the Impact of the $2 \mathrm{D} / 3 \mathrm{D}$ Structures on Performance and Stability of Perovskite Solar Cells. Sol. RRL 2019.

(8) Cho, K. T.; Zhang, Y.; Orlandi, S.; Cavazzini, M.; Zimmermann, I.; Lesch, A.; Tabet, N.; Pozzi, G.; Grancini, G.; Nazeeruddin, M. K. Water-Repellent Low-Dimensional Fluorous Perovskite as Interfacial Coating for $20 \%$ Efficient Solar Cells. Nano Lett. 2018, 18 (9), 5467-5474.

(9) Proppe, A. H.; Quintero-Bermudez, R.; Tan, H.; Voznyy, O.; Kelley, S. O.; Sargent, E. H. Synthetic Control over Quantum Well Width Distribution and Carrier Migration in Low-Dimensional Perovskite Photovoltaics. J. Am. Chem. Soc. 2018, 140 (8), 2890-2896.

(10) Tsai, H.; Nie, W.; Blancon, J.-C.; Stoumpos, C. C.; Asadpour, R.; Harutyunyan, B.; Neukirch, A. J.; Verduzco, R.; Crochet, J. J.; Tretiak, S.; et al. High-Efficiency Two-Dimensional Ruddlesden-Popper Perovskite Solar Cells. Nature 2016, 536 (7616), 312-316.

(11) Stoumpos, C. C.; Soe, C. M. M.; Tsai, H.; Nie, W.; Blancon, J.-C.; Cao, D. H.; Liu, F.; Traoré, B.; Katan, C.; Even, J.; et al. High Members of the 2D Ruddlesden-Popper Halide Perovskites: Synthesis, Optical Properties, and Solar Cells of $\left(\mathrm{CH}_{3}\left(\mathrm{CH}_{2}\right)_{3} \mathrm{NH}_{3}\right)_{2}\left(\mathrm{CH}_{3} \mathrm{NH}_{3}\right)_{4} \mathrm{~Pb}_{5} \mathrm{I}_{16}$. Chem 2017, 2 (3), 427440 .

(12) Zhang, X.; Wu, G.; Fu, W.; Qin, M.; Yang, W.; Yan, J.; Zhang, Z.; Lu, X.; Chen, H. Orientation Regulation of Phenylethylammonium Cation Based 2D Perovskite Solar Cell with Efficiency Higher Than $11 \%$. Adv. Energy Mater. 2018, 8 (14), 1702498.

(13) Yang, X.; Zhang, X.; Deng, J.; Chu, Z.; Jiang, Q.; Meng, J.; Wang, P.; Zhang, L.; Yin, Z.; You, J. Efficient Green Light- 
Emitting Diodes Based on Quasi-Two-Dimensional Composition and Phase Engineered Perovskite with Surface Passivation. Nat. Commun. 2018, 9 (1), 570.

(14) Tsai, H.; Nie, W.; Blancon, J.-C.; Stoumpos, C. C.; Soe, C. M. M.; Yoo, J.; Crochet, J.; Tretiak, S.; Even, J.; Sadhanala, A.; et al. Stable Light-Emitting Diodes Using Phase-Pure Ruddlesden-Popper Layered Perovskites. Adv. Mater. 2018, 30 (6), 1704217.

(15) Li, J.; Wang, J.; Ma, J.; Shen, H.; Li, L.; Duan, X.; Li, D. SelfTrapped State Enabled Filterless Narrowband Photodetections in 2D Layered Perovskite Single Crystals. Nat. Commun. 2019, 10 (1), 806.

(16) Stoumpos, C. C.; Cao, D. H.; Clark, D. J.; Young, J.; Rondinelli, J. M.; Jang, J. I.; Hupp, J. T.; Kanatzidis, M. G. Ruddlesden-Popper Hybrid Lead Iodide Perovskite 2D Homologous Semiconductors. Chem. Mater. 2016, 28 (8), 2852-2867.

(17) Smith, M. D.; Connor, B. A.; Karunadasa, H. I. Tuning the Luminescence of Layered Halide Perovskites. Chem. Rev. 2019, 119 (5), 3104-3139.

(18) Wang, Z.; Lin, Q.; Chmiel, F. P.; Sakai, N.; Herz, L. M.; Snaith, H. J. Efficient Ambient-Air-Stable Solar Cells with 2D-3D Heterostructured Butylammonium-CaesiumFormamidinium Lead Halide Perovskites. Nat. Energy 2017, 2 (9), 17135.

(19) Grancini, G.; Roldán-Carmona, C.; Zimmermann, I.; Mosconi, E.; Lee, X.; Martineau, D.; Narbey, S.; Oswald, F.; De Angelis, F.; Graetzel, M.; et al. One-Year Stable Perovskite Solar Cells by 2D/3D Interface Engineering. Nat. Commun. 2017, 8 (1), 15684.

(20) Even, J.; Pedesseau, L.; Katan, C. Understanding Quantum Confinement of Charge Carriers in Layered 2D Hybrid Perovskites. ChemPhysChem 2014, 15 (17), 3733-3741.

(21) Straus, D. B.; Kagan, C. R. Electrons, Excitons, and Phonons in Two-Dimensional Hybrid Perovskites: Connecting Structural, Optical, and Electronic Properties. J. Phys. Chem. Lett. 2018, 9 (6), 1434-1447.

(22) Blancon, J.-C.; Tsai, H.; Nie, W.; Stoumpos, C. C.; Pedesseau, L.; Katan, C.; Kepenekian, M.; Soe, C. M. M.; Appavoo, K.; Sfeir, M. Y.; et al. Extremely Efficient Internal Exciton Dissociation through Edge States in Layered 2D Perovskites. Science (80-. ). 2017, 355 (6331), 1288-1292.

(23) Chen, Y.; Sun, Y.; Peng, J.; Tang, J.; Zheng, K.; Liang, Z. 2D Ruddlesden-Popper Perovskites for Optoelectronics. Adv. Mater. 2018, 30 (2), 1703487.

(24) Mao, L.; Stoumpos, C. C.; Kanatzidis, M. G. TwoDimensional Hybrid Halide Perovskites: Principles and Promises. J. Am. Chem. Soc. 2019, 141 (3), 1171-119o.

(25) Smith, I. C.; Hoke, E. T.; Solis-Ibarra, D.; McGehee, M. D.; Karunadasa, H. I. A Layered Hybrid Perovskite Solar-Cell Absorber with Enhanced Moisture Stability. Angew. Chemie 2014, 126 (42), 11414-11417.

(26) Quintero-Bermudez, R.; Gold-Parker, A.; Proppe, A. H.; Munir, R.; Yang, Z.; Kelley, S. O.; Amassian, A.; Toney, M. F.; Sargent, E. H. Compositional and Orientational Control in Metal Halide Perovskites of Reduced Dimensionality. Nat. Mater. 2018, 17 (10), 900-907.

(27) Jemli, K.; Audebert, P.; Galmiche, L.; Trippé-Allard, G.; Garrot, D.; Lauret, J. S.; Deleporte, E. Two-Dimensional Perovskite Activation with an Organic Luminophore. ACS Appl. Mater. Interfaces 2015, 7 (39), 21763-21769.

(28) Herckens, R.; Van Gompel, W. T. M.; Song, W.; GélvezRueda, M. C.; Maufort, A.; Ruttens, B.; D'Haen, J.; Grozema, F. C.; Aernouts, T.; Lutsen, L.; et al. Multi-Layered Hybrid Perovskites Templated with Carbazole Derivatives: Optical Properties, Enhanced Moisture Stability and Solar Cell Characteristics. J. Mater. Chem. A 2018, 6 (45), 2289922908.

(29) Mitzi, D. B. Synthesis, Crystal Structure, and Optical and Thermal Properties of $\left(\mathrm{C}_{4} \mathrm{H}_{9} \mathrm{NH}_{3}\right)_{2} \mathrm{MI}_{4}(\mathrm{M}=\mathrm{Ge}, \mathrm{Sn}, \mathrm{Pb})$. Chem. Mater. 1996, 8 (3), 791-80o.
(30) Yangui, A.; Garrot, D.; Lauret, J. S.; Lusson, A.; Bouchez, G.; Deleporte, E.; Pillet, S.; Bendeif, E. E.; Castro, M.; Triki, S.; et al. Optical Investigation of Broadband White-Light Emission in Self-Assembled Organic-Inorganic Perovskite $\left(\mathrm{C}_{6} \mathrm{H}_{11} \mathrm{NH}_{3}\right)_{2} \mathrm{PbBr}_{4}$. J. Phys. Chem. C 2015, 119 (41), 2363823647.

(31) Dohner, E. R.; Jaffe, A.; Bradshaw, L. R.; Karunadasa, H. I. Intrinsic White-Light Emission from Layered Hybrid Perovskites. J. Am. Chem. Soc. 2014, 136 (38), 13154-13157.

(32) Billing, D. G.; Lemmerer, A. Synthesis, Characterization and Phase Transitions in the Inorganic-Organic Layered Perovskite-Type Hybrids $\left[\left(\mathrm{C}_{\mathrm{n}} \mathrm{H}_{2 \mathrm{n}+} \mathrm{NH}_{3}\right)_{2} \mathrm{PbI}_{4}\right], \mathrm{n}=4,5$ and 6. Acta Crystallogr. Sect. B Struct. Sci. 20o7, 63 (5), 735-747.

(33) Mousdis, G. A.; Papavassiliou, G. C.; Raptopoulou, C. P.; Terzis, A. Preparation and Characterization of $\left[\mathrm{H}_{3} \mathrm{~N}\left(\mathrm{CH}_{2}\right)_{6} \mathrm{NH}_{3}\right] \mathrm{PbI}_{4}$ and Similar Compounds with a Layered Perovskite Structure. J. Mater. Chem. 20oo, 10 (2), 515-518.

（34） Yuan, Z.; Shu, Y.; Tian, Y.; Xin, Y.; Ma, B. A Facile One-Pot Synthesis of Deep Blue Luminescent Lead Bromide Perovskite Microdisks. Chem. Commun. 2015, 51 (91), 1638516388.

(35) Weidman, M. C.; Seitz, M.; Stranks, S. D.; Tisdale, W. A. Highly Tunable Colloidal Perovskite Nanoplatelets through Variable Cation, Metal, and Halide Composition. ACS Nano 2016, 10 (8), 7830-7839.

(36) Bekenstein, Y.; Koscher, B. A.; Eaton, S. W.; Yang, P.; Alivisatos, A. P. Highly Luminescent Colloidal Nanoplates of Perovskite Cesium Lead Halide and Their Oriented Assemblies. J. Am. Chem. Soc. 2015, 137 (51), 16008-16011.

(37) Sheng, X.; Chen, G.; Wang, C.; Wang, W.; Hui, J.; Zhang, Q.; Yu, K.; Wei, W.; Yi, M.; Zhang, M.; et al. Polarized Optoelectronics of $\mathrm{CsPbX}_{3}(\mathrm{X}=\mathrm{Cl}, \mathrm{Br}, \mathrm{I})$ Perovskite Nanoplates with Tunable Size and Thickness. Adv. Funct. Mater. 2018, 28 (19), 1800283.

(38) Akkerman, Q. A.; Motti, S. G.; Srimath Kandada, A. R.; Mosconi, E.; D’Innocenzo, V.; Bertoni, G.; Marras, S.; Kamino, B. A.; Miranda, L.; De Angelis, F.; et al. Solution Synthesis Approach to Colloidal Cesium Lead Halide Perovskite Nanoplatelets with Monolayer-Level Thickness Control. J. Am. Chem. Soc. 2016, 138 (3), 1010-1016.

(39) Akkerman, Q. A.; Rainò, G.; Kovalenko, M. V.; Manna, L. Genesis, Challenges and Opportunities for Colloidal Lead Halide Perovskite Nanocrystals. Nat. Mater. 2018, 17 (5), 394-405.

(40) Shamsi, J.; Urban, A. S.; Imran, M.; De Trizio, L.; Manna, L. Metal Halide Perovskite Nanocrystals: Synthesis, PostSynthesis Modifications, and Their Optical Properties. Chem. Rev. 2019, 119 (5), 3296-3348.

(41) Almeida, G.; Goldoni, L.; Akkerman, Q.; Dang, Z.; Khan, A. H.; Marras, S.; Moreels, I.; Manna, L. Role of Acid-Base Equilibria in the Size, Shape, and Phase Control of Cesium Lead Bromide Nanocrystals. ACS Nano 2018, 12 (2), 17041711.

(42) Maceiczyk, R. M.; Dümbgen, K.; Lignos, I.; Protesescu, L.; Kovalenko, M. V.; DeMello, A. J. Microfluidic Reactors Provide Preparative and Mechanistic Insights into the Synthesis of Formamidinium Lead Halide Perovskite Nanocrystals. Chem. Mater. 2017, 29 (19), 8433-8439.

(43) Langford, J. I.; Wilson, A. J. C. Scherrer after Sixty Years: A Survey and Some New Results in the Determination of Crystallite Size. J. Appl. Crystallogr. 1978, 11 (2), 102-113.

(44) Ishihara, T. Optical Properties of PbI-Based Perovskite Structures. J. Lumin. 1994, 6o-61, 269-274.

(45) Iwanaga, M.; Azuma, J.; Shirai, M.; Tanaka, K.; Hayashi, T. Self-Trapped Electrons and Holes in $\mathrm{PbBr}_{2}$ Crystals. Phys. Rev. B 2002, 65 (21), 214306.

(46) Wu, X.; Trinh, M. T.; Niesner, D.; Zhu, H.; Norman, Z.; Owen, J. S.; Yaffe, O.; Kudisch, B. J.; Zhu, X.-Y. Trap States in Lead Iodide Perovskites. J. Am. Chem. Soc. 2015, 137 (5), 2089-2096. 
